\title{
THE AGS BOOSTER BEAM POSITION MONITOR SYSTEM"
}

\author{
D.J. Ciardullo, A. Abola, E.R. Beadle, G.A. Smith, R. Thomas, \\ W. Van Zwienen, R. Warkentien, R.L. Witkover \\ BNL-- 45418 \\ Brookhaven National Laboratory \\ Upton, New York 11973

\section{Abstract}

To accelerate both protons and heavy ions, the AGS Booster requires a broadband (multi-octave) beam position monitoring system with a dynamic range spanning several orders of magnitude $\left(2 \times 10^{10}\right.$ to $1.5 \times 10^{13}$ particles per pulse). System requirements include the ability to acquire single tum trajectory and average orbit information with \pm 0.1 $\mathrm{mm}$ resolution. The design goal of $\pm 0.5 \mathrm{~mm}$ corrected eccuracy requires that the detectors have repeatable linear performance after periodic bakeout at $300^{\circ} \mathrm{C}$. The system design and capabilities of the Booster Beam Position Monitor will be described, and initial results presented.

\section{Introduction}

The measurement parameters for this system are [1]:
Absolute accuracy:
$\pm 0.5 \mathrm{~mm}$
Precision:
$\pm 0.1 \mathrm{~mm}$
Circular aperture:

Frequency range:

Intensity renge:

Bunch width (at half $\max$ ):

$0.2 \times 4.11 \mathrm{MHz}$

$2 \times 10^{10}$ to $1.5 \times 10^{13} \mathrm{ppp}$

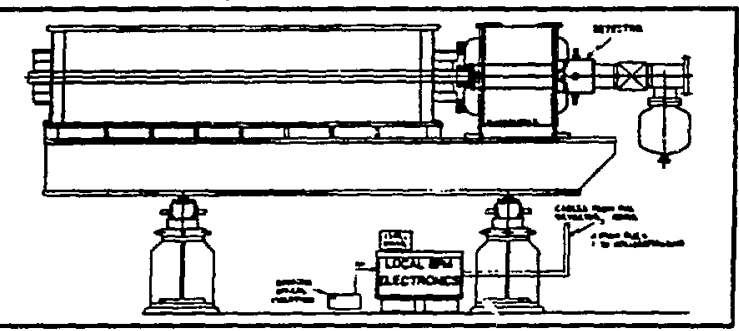

Fig. 2 A Typical Booster BPM Station.

Figure 1 is a block diagram of the AGS Booster BPM system. There are $46 \mathrm{BPM}$ stations within the Booster ring, each responsible for average orbit measurement in a single plane

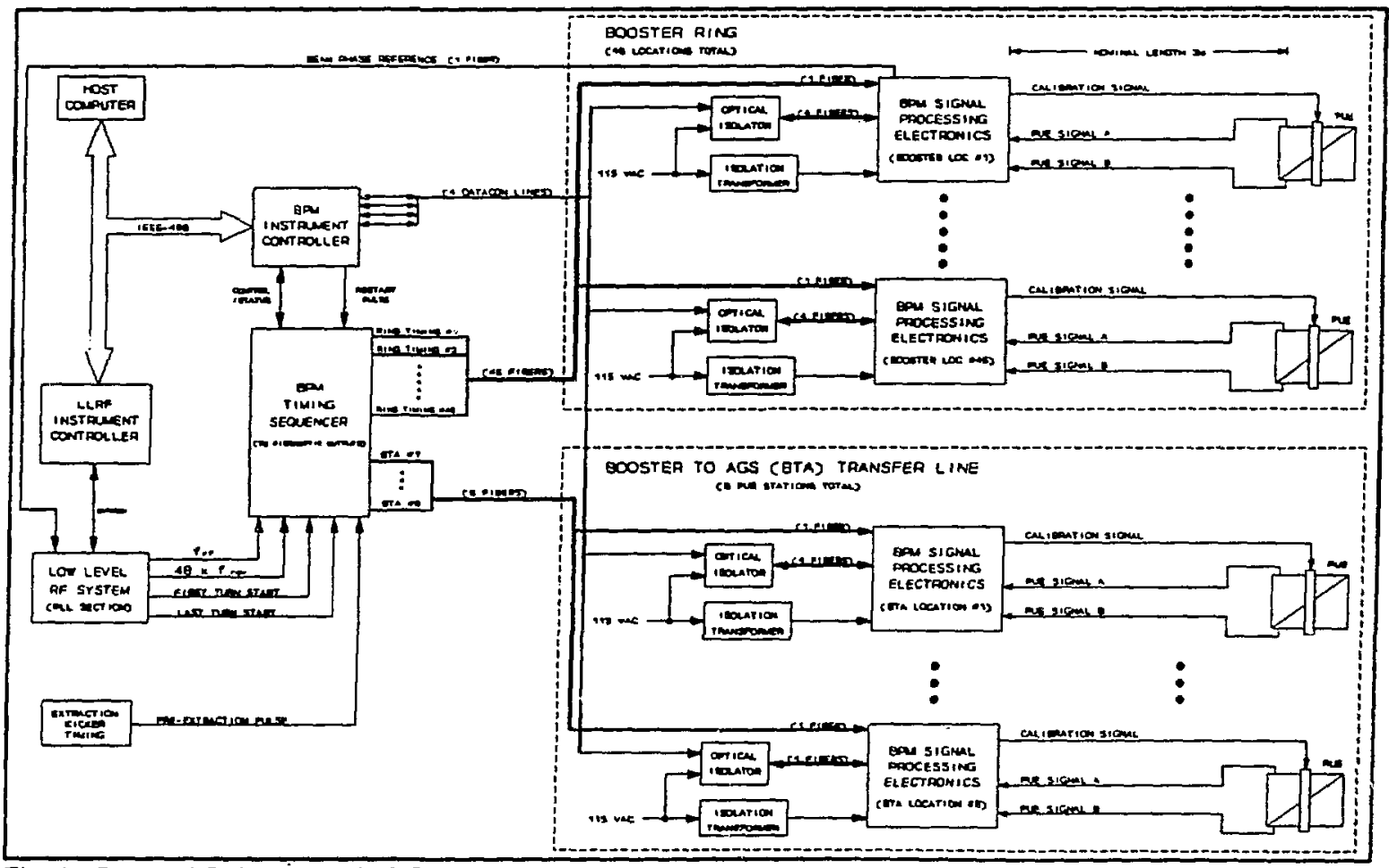

Fig. 1 Booster BPM System Block Diagram.

- Work performed under the auspices of the U.S. Department Of Energy. 
(22 horizontal and 24 vertical). Six additional stations are located in the Booster To AGS (BTA) transfer line. At each station there is a beam sensor located at the appropriate betamax and locked to a quadrupole on the central orbit. Signals from the detector go to processing and acquisition hardware, located on the floor of the Booster just below each quadrupole (Figure 2). Digitized information is retrieved from exch station up to six times per Booster cycle. The system provides average orbit information (over up to 80 turns) or first turn trajectory. In addition, analog real-time outputs of either the bunch signals or their sum and difference are available from any location for use by other systems.

\section{The Derector}

The beam sensor is the split cylinder electrostatic type, chosen for its flat low frequency response and linear induced electrode signal vs. beam position. Ench detector has been tested prior to its installation in the Booster, and has a verified linearity and resolution of greater than $\pm 0.1 \mathrm{~mm}$ over the 60 $\mathrm{mm}$ circular measurement aperture [2]. A built-in calibration ring couples equally to both pick up electrodes (PUEs), simulating a centered beam when a test signal is applied from the electronics. This ran be used to obtain a value for the total system offset, referenced to beampipe center. There are four If N-type vacuum feedthrus which bring signals into and out of the detector; two for the calibration ring (one of which is terminated with 50 ohms), and one for each PUE of th split cylinder.

\section{Signal Cable Assemblies}

A 3 meter length of standard 93 ohm coexial cable, modified to have a solid shield, is used to carry the PUE signals from the detector to the processing electronics (Fig. 3).

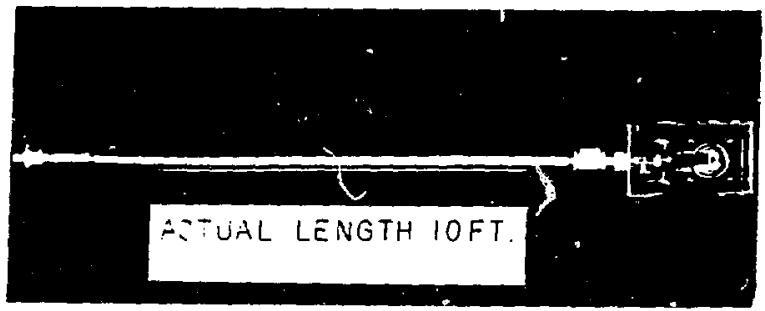

Fig. 3 Mock-up of PUE Signal Cab!e Assembly.

The length of the cable is a compromise between several factors. The main objective was to locate the processing electronics far enough away from the beamline to minimize radiation damage. The lingth must be limited, however, to reduce signal loss due to capacitive loading of the PUEs, and to prevent excessive peaking at the top end of the bunch signal response. A 200 ohm resistor (at the PUE end of the assembly) belps to flatten this peaking at the high end of the processing bandwidth (approximately $25 \mathrm{MHz}$ ). RG-62 coaxial cable was chosen for its low cost, moderate capacitance per unit length $(46 \mathrm{pF} / \mathrm{m})$ and reasonable radiation survivability (semi-solid polyethylene). The cable is enclosed by standard $3 / 8$ inch copper tubing, which is electrically connected to the shield at both ends of the assembly. Each assembly is measured on a nerwork analyzer, and units are paired by matching their responses to within $\pm 0.01 \mathrm{~dB}$ (to $30 \mathrm{MHz}$ ).

The use of Thermal Isolators at each of the four vacuum feedthrus on the detector allows signal cables to remain connected during bakeout at $300^{\circ} \mathrm{C}$. The isolators are effectively sf coarial gir lines, $14 \mathrm{~cm}$ in length, with male $\mathrm{N}$ type connectors on either end. This length is negligible as compared to the processing bandwidth of the electronics, and adds only $8 \mathrm{pF}$ to the total capacitance load on each PUE.

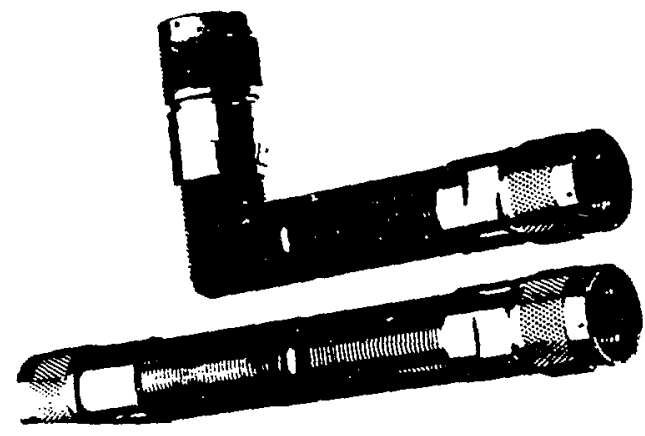

Fig. 4 Two versions of the Thermal Isolator.

Stainless steel construction provides a temperature drop (at the center conductor, $25^{\circ} \mathrm{C}$ ambient) from $200^{\circ} \mathrm{C}$ to approximately $60^{\circ} \mathrm{C}$. In those areas with mechanical obstructions near the beampipe, an angled version of the Thermal Isolator is used instead. Capacitance variations between assemblies are less than $\pm 0.1 \mathrm{pF}$.

\section{Local BPM Signal Processing Electronics}

The Local Signal Processing crate is built into a Eurocard style subrack for modularity (Figure 5). The body of the subrack is isolated from ground and allowed to float at beampipe

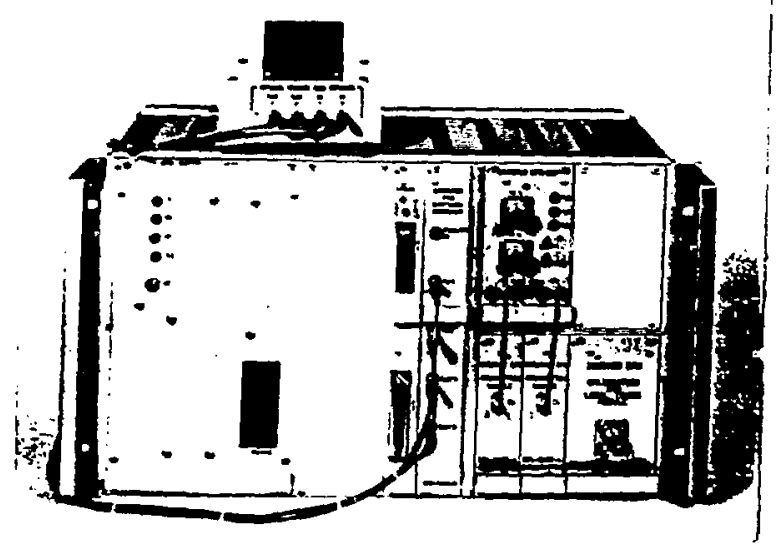

Fig. 5 Local BPM Processing Electronics. 
potential for purposes of increased noise immunity. The top right slot is reserved for a dual analog fiberoptic transmitter module, used to isolate the electronics from any external systems requiring beam position information. A Test Access Panel (TAP) is included in each crate as a maintainence aide. This card allows accessibility to backplane signals, and has provision for switching the crate to local control.

The local processing electronics are divided into three major sections [3]; Front End Processing (FEP), Acquisition (ACQ) and Calibration and LOcal Timing (CLT). The FEP module provides appropriate PUE signal scaling and frequency compensation. Both channels of the FEP are matched to within $0.02 \mathrm{~dB}$ (to $20 \mathrm{MHz}$ ) in all three gain modes, resulting in a position accuracy for this module of better than $0.3 \mathrm{~mm}$ A $\pm 1 \mathrm{~dB}$ flatmess preserves the temporal response of the PUE signals for bunch widths down to 50 ns (haif max) [4]. Each module hes a secondary set of outputs which provides either buffered PUE signals or their sum and difference. These can be used for diagnostic purposes, or as real-time position information by other systems.

Two Acquisition ( $A C Q$ ) modules and the Calibration and Local Timing (CLD) module form the next PUE signal processing step. Sum and difference signals from the FEP are baseline restored (the capacitively coupled PUE signals have zero average value), integrated then digitized by the ACQ. Local timing and control signals are generated by the CLT module. This module receives a properly phased pulse burst from the Sequencer and generates the BLR, integrate gating and reset pulses for each ACQ. The number of beam buzches over which to integrate can be selected from 1 to 255 (up to 85 tums), and the resulting integrals are digitized to 13 bits. In addition, the CLT module can generate pulses to drive the calibration ring of the detector at five selectable frequencies. This can be used to check system connections and to measure total system offset errors [5].

The Controller Interface Electronics (CIE) card is the local interface between the crate and the BPM lnstrument Controller. This boand passes digital data, control and status signals to and from the Controller via a fiberoptic version of DATACON which eliminates ground loops and allows the crate to floas at beampipe potential. The CIE card also serves as the fiberoptic interface between the Sequencer and the CLT module.

\section{Sequencer}

The Sequencer is located remole from the Booster ring and connected to each of the 52 BPM stations via digital fiberoptic link [6]. The Booster Low Level RF (LLRF) system supplies the Sequencer with $f_{r f}$ and $48 \times f_{k v v}$ both of which are phase locked to the beam [7] (beam phase information is fed back to the LLRF system from the secondary sum output of an FEP module in the ring). These two signals are used to generate a burst (of specified duration) of BLR pulses for use by the Calibration and Local Timing module at each BPM station. The BLR pulses are phased such that they always occur between bunches, independent of harmonic number. The LLRF system also supplies the Sequencer with triggers which indicate the start of the first and last urns in the Booster cycle. Since the BPM stations in the extraction line (BTA) always see three bunches at the end of the acceleration cycle, no baseline restoration of the sum and difference signals are necessary before integration. For the six BPM stations in the BTA line, the Sequencer always outputs a burst of three BLR pulses.

\section{High Level Software Control}

The BPM Instrument Controller interfaces the host computer (Apollo system) with the Sequencer and the local processing electronics, and provides reports to the software of PUE sum and difference daka from one or more samples during the Booster cycle.

The "booster_orbit" program is the high level applications software for the Beam Position Monitor, running on the Apollo computer system. Its primary function is to graphically represent the equilibrium orbit of the Booster ring at any time during the cycle. The program allows for the acquisition of data with varying parameters such as number of turns (over which to integrate), number of BPM Booster groups $w$ acquire information from, acquisition times and gain settings. The program also allows for a variety of displays using saved, current or difference orbits with varying modes of averaging and plotting parameters. Statistical display options are also available.

In addition to orbit acquisition and display, the scope of the program extends to diagnostic testing of the hardware, full calibration of each of the local BPM stations and display/update of static and dynamic BPM parameters (e.g. values of differential sensitivity, system offsets and operational status) used in the position calculations.

\section{Acknowledgement}

The authors wish to express their gratitude to J.M. Brennan for the considerable conceptual advice and expertise he has contributed throughout the course of this project.

[1] E. Beadle, et al, "Design of the AGS Booster Beam Position Monitor System, "Procedings of the 1089 IEEE Particle Accelerator Conference.

[2] R. Thomas, et al, "Design and Testing of the AGS Booster BPM Detertor," this conference.

[3] D.J. Ciardullo, et al, "Design of the AGS Booster Beam Position Monitor Electronics," this conference.

[4] D.J. Ciardullo, private communication.

[5] D.J. Ciardullo, private communication.

[6] op cit.

[7] T. Hayes, A. Zaitsman "Indirect Phase Locking of RF Clock to the Beam for the BNL Booster BPM Sysiem," this conference. 